\title{
A multiscale model for the rupture of linear polymers in strong flows
}

\author{
E. Rognin ${ }^{1} \dagger$, N. Willis-Fox ${ }^{1}$, T. A. Aljohani ${ }^{2}$, R. Daly ${ }^{1}$ \\ ${ }^{1}$ Institute for Manufacturing, Department of Engineering, University of Cambridge, 17 Charles \\ Babbage Road, Cambridge, CB30FS, United Kingdom \\ ${ }^{2}$ King Abdulaziz City for Science and Technology, P.O Box 6086, Riyadh 11442, Kingdom of Saudi \\ Arabia
}

(Received xx; revised xx; accepted xx)

Polymer-containing solutions used across research and industry are commonly exposed to mechanically harsh fluid processes, for example shear and extensional forces during flow through porous media or rapid micro-dispensing of biopharmaceutical molecules. These forces are strong enough to break the covalent bonds in the polymer backbone. As this scission phenomenon can change the functional and fluid-flow properties as well as introduce reactive radicals into the solution, it must be understood and controlled. Experiments and models to-date have only provided partial or qualitative insights into this behaviour. Here we build a link between the molecular-scale degradation models and the macro-scale laminar flow of dilute solutions in any given geometry. A free-draining bead-rod model is used to investigate rupture events at the molecular scale. It is shown by uniaxial extension simulations of an ensemble of chains that scission can be conveniently described at the macroscopic scale as a first order reaction whose rate is a function of the conformation tensor of the macromolecules and the velocity gradient of the flow. This approach is implemented in the finite volume code OpenFOAM by elaborating an appropriate constitutive equation for the conformation tensor. The macroscopic model is run and analysed for ultra-dilute solutions of poly(methyl methacrylate) in ethyl acetate and polyethylene oxide in water, using the geometry of an abrupt contraction flow and neglecting any viscoelastic effect. This multiscale approach bridges the gap between phenomenological observations of mechanicallyinduced chemical degradation in large scale applications and the rich field of molecularscale models of macromolecules under flow.

\section{Introduction}

Covalent bonds found in macromolecule backbones can break due to strong elongational flows (May \& Moore 2013). This phenomenon is of particular importance in both industrial and research environments, such as the use and degradation over time of drag-reducing polymers for oil recovery and fluid conveying (Lumley 1969; Paterson \& Abernathy 1970; Barnard \& Sellin 1972; Virk 1975; Choi et al. 2000; Elbing et al. 2011; Pereira \& Soares 2012), the mechanical fragmentation of DNA for high throughput gene sequencing (Thorstenson et al. 1998; Shendure \& Ji 2008; Grokhovsky et al. 2011), or the preservation of plasmid DNA or other fragile biopharmaceutical macromolecules throughout their manufacturing process (Prather et al. 2003; Rathore \& Rajan 2008; Wu et al. 2009; Hawe et al. 2012). In addition, molecules under tension in strong flows have received significant attention over the past decade from the field of mechanochemistry-the science of chemical reactions brought about by mechanical energy (Caruso et al. 2009; Li et al. 2015). As a matter of fact,

$\dagger$ Email address for correspondence: ecr43@cam.ac.uk 
mechanically halving polymer chains yields free radicals that can then feed more elaborate reactions, opening up new opportunities in materials science and manufacturing.

Regarding polymer scission in fluid flows, a great deal of work has been carried out to understand the rupture dynamics and its key parameters, such as shear and extensional rates in the flow, viscosity and quality of the solvent, type of polymer, molecular weight and concentration (for a comprehensive review, see May \& Moore 2013). In spite of numerous experiments, theoretical approaches, and simulations, comparisons between different setups or flow patterns can still prove to be puzzling because of the complex nature of the coupling between macroscopic parameters and molecular scission. For example, the flow gradients in a sonicated bath cannot be known accurately due to the highly transient and heterogeneous locations of cavitation bubbles, and the dependence of their dynamics on the sonication device, the vial geometry and the polymer solution itself. For dilute polymer solutions in turbulent flows, Vanapalli et al. (2006) succeeded in deriving a scaling law for the critical shear rate (deformation rate of the flow above which a given molecular weight is broken) according to the contour length of the molecule and the Reynolds number of the flow. Their scaling law accounts for experimental data of four kinds of polymer-polystyrene (PS), polyoxyethylene (PEO), polyacrylamide (PAM) and DNA-over a range of Reynolds number of several orders of magnitude. One striking feature of their model is that the onset of rupture only depends on the amplitude of strain rates at the small viscous scale (or Kolmogorov scale) and not on macroscopic flow patterns. Nevertheless, no quantitative information is given on the rate of scission. Moreover, at moderate Reynolds numbers where the flow is laminar or the turbulence not fully developed, the geometry of the flow is likely to play a role since high strain regions are concentrated near walls and contractions. Such situations can be encountered in microfluidics (Kang et al. 2005; Nghe et al. 2010), flows through porous media (James \& Mclaren 1975), or precision dispensing processes like inkjet printing where polymer scission has been reported (A-Alamry et al. 2011).

Therefore, a general model for polymer rupture at the scale of the fluid process is still lacking. Scientists and engineers could however benefit from a macroscopic computational approach to efficiently prevent chain scission when trying to protect delicate molecules, or design new manufacturing processes based on mechanochemistry. Here, we present the implementation of a chain scission model in computational fluid dynamics simulations (CFD), with the help of coarse-grained molecular simulations.

The paper is organised as follows: in the next section, we present our modelling approach starting by a review of mechanical bond rupture simulations from the atomic scale to the macroscopic scale. Then we go through preliminary theoretical work and simulations, using the bead-rod model as a coarse-grained molecular model of a linear polymer. A formalism for the macroscopic scale is then derived and tested against various microscopic parameters. Finally, the CFD model is tested for the case of an abrupt contraction flow. Some concluding remarks are drawn about the relevance and perspectives of this method.

\section{General approach}

Broadly speaking, simulations of mechanochemistry can be categorised into four different methods, each relevant to a range of length and time scales, as illustrated in figure 1 . The smallest length scales and time scales are explored using tools of quantum chemistry or ab initio molecular dynamics. The idea is to solve the electronic structure of a small molecule under a given strain and derive which bond would break and how much force would be involved (Stauch \& Dreuw 2016). The next level called all-atom molecular dynamics solves the motion of molecules (polymer and solvent) described by their constituent atoms, and determines the stretching and vibration of the bonds, according to classical mechanics and 


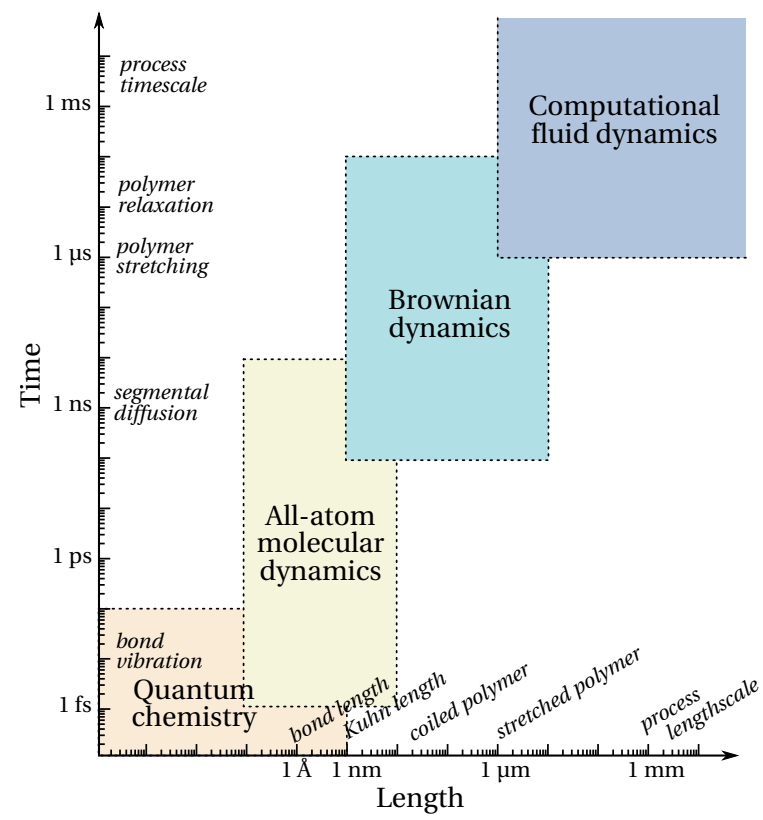

Figure 1: Scales involved in different methods of simulation.

pre-set force fields between atoms. This approach can be used to study how stress propagates though the bonds of the backbone of a long molecule (Stacklies et al. 2009; RibasArino \& Marx 2012). Intermediary length and time scales are analysed using coarse-grained molecular models, including the bead-rod and bead-spring models. In this approach, the solvent is considered as a continuous medium applying hydrodynamic forces on beads connected together by rigid rods or elastic springs, respectively. The molecular nature of this physics called Brownian dynamics, is simulated by adding a random noise to the fluid flow, accounting for sporadic collisions of solvent molecules. These models have been used to study the build-up of internal tension caused by extensional flows (López Cascales \& García de la Torre 1992; Knudsen et al. 1996b; Maroja et al. 2001; Hsieh et al. 2005; Sim et al. 2007). The simplicity of these representations allows longer time scales and multiple runs to derive ensemble-averaged quantities. Finally, the coarsest level of time and length scales is a continuous mechanics and mass transfer approach. The polymer population is described by its concentration, and the mechanochemistry is solved in terms of reaction rates and convection of the products by the fluid flow (Bestul 1956; López Cascales \& García de la Torre 1992; Reese \& Zimm 1990). Here, we explore in details the links between the coarse-grained molecular models and the continuous scale.

For many years, researchers have developed rheological models to compute the mechanical stress caused by the presence of macromolecules in flowing solutions or melts (Bird et al. 1987; Bird \& Wiest 1995; Van Den Brule, B. et al. 2011; Larson \& Desai 2015). A specific class of tools, called constitutive equations, are used to express the viscoelastic stress in terms of the kinematics tensors of the flow and additional state variables. For constitutive equations inspired by molecular models, one option is to describe the polymer population by the conformation tensor, $\boldsymbol{A}$, defined as the ensemble average of the tensorial product of the individual end-to-end vectors, $\boldsymbol{R}$, (often written $\boldsymbol{A}=\langle\boldsymbol{R} \boldsymbol{R}\rangle$ ) (Rallison \& Hinch 1988). Higher order moments can be employed in more sophisticated representations (Lielens et al. 1999; Ilg et al. 2010). 


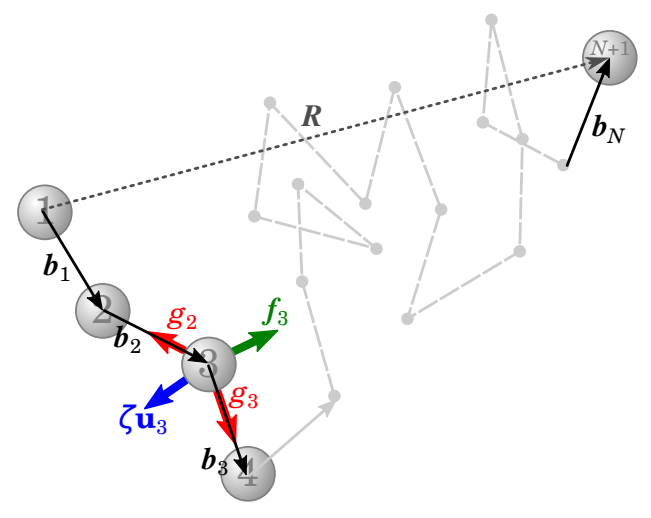

Figure 2: Bead-rod model for a polymer chain.

Although coarse-grained molecular models have been an important source of inspiration for mechanical stress equations, no link has previously been made for the phenomenon of polymer fracture, in contrast with the field of flow-induced scission of micelles where macroscopic models have been developed (Vasquez et al. 2007). The core idea of this study is then to compute the internal tension in polymer chains and backbone scission rate by relying on the conformation tensor and the fluid velocity gradient. In the next section, we describe the steps needed to achieve this modelling framework.

\section{Model}

\subsection{The free-draining bead-rod model}

Following the work of Sim et al. (2007), we employ the free-draining bead-rod model as our coarse-grained molecular approach. A complete description of this model may be found in Bird et al. (1987); Liu (1989); Doyle et al. (1997); Sim et al. (2007); we present here its main characteristics. The polymer chain is represented by a series of $N$ rigid rods connecting $N+1$ beads (see figure 2). The rods (vectors $\boldsymbol{b}_{1}, \ldots, \boldsymbol{b}_{N}$ ) are free to rotate but must keep a constant length $b$, known as the Kuhn length. The sum of the rods give the end-to-end vector, $\boldsymbol{R}$. The equation of motion for the bead $i$ is given by:

$$
\zeta \boldsymbol{v}_{i}=\zeta \boldsymbol{u}_{i}+g_{i} \boldsymbol{r}_{i}-g_{i-1} \boldsymbol{r}_{i-1}+\boldsymbol{f}_{i},
$$

where $\zeta$ is the friction coefficient of the bead with the solvent, $\boldsymbol{u}_{i}$ is the unperturbed fluid velocity at the location of the bead (velocity field in absence of the chain), $g_{i}$ and $g_{i-1}$ are the internal tensions directed along the directions of the rods $\boldsymbol{r}_{i}=\boldsymbol{b}_{i} / b$ and $\boldsymbol{r}_{i-1}=\boldsymbol{b}_{i-1} / b$ (except at both ends of the chain where there is only one tension vector), and $\boldsymbol{f}_{i}$ a stochastic force modelling Brownian diffusion motion. The $\boldsymbol{f}_{i}$ are uncorrelated in space and time, of zero average and variance $2 k_{B} T \zeta$ for each space coordinate, $k_{B} T$ being the thermal energy. The evolution equation for each rod is then:

$$
\frac{\mathrm{d} \boldsymbol{b}_{i}}{\mathrm{~d} t}=\boldsymbol{v}_{i+1}-\boldsymbol{v}_{i}
$$


Integrating with an explicit Euler scheme over a time step $\delta t$ while enforcing the rod length to remain constant leads to the following system of equations for the internal tensions:

$$
-g_{i+1} \boldsymbol{r}_{i} \cdot \boldsymbol{r}_{i+1}+2 g_{i}-g_{i-1} \boldsymbol{r}_{i} \cdot \boldsymbol{r}_{i-1}=\zeta b \boldsymbol{r}_{i} \cdot \boldsymbol{K} \cdot \boldsymbol{r}_{i}+\boldsymbol{r}_{i} \cdot\left(\boldsymbol{w}_{i+1}-\boldsymbol{w}_{i}\right)+\frac{\zeta \delta t}{2 b}\left(\boldsymbol{v}_{i+1}-\boldsymbol{v}_{i}\right)^{2}
$$

with $\boldsymbol{K}=\boldsymbol{\nabla} \boldsymbol{u}^{T}$ is the transpose of the fluid velocity gradient and $\boldsymbol{w}_{i}$ are random vectors of mean zero and variance $2 k_{B} T \zeta / \delta t$. The last term is non-linear and is iteratively solved by a fixed point method.

Equation 3.3 can be made dimensionless by dividing forces by $k_{B} T / b$, lengths by $b$ and time by $\zeta b^{2} /\left(k_{B} T\right)$ :

$$
-g_{i+1}^{+} \boldsymbol{r}_{i} \cdot \boldsymbol{r}_{i+1}+2 g_{i}^{+}-g_{i-1}^{+} \boldsymbol{r}_{i} \cdot \boldsymbol{r}_{i-1}=\operatorname{Pe} \boldsymbol{r}_{i} \cdot \boldsymbol{K} \cdot \boldsymbol{r}_{i}+\boldsymbol{r}_{i} \cdot\left(\boldsymbol{w}_{i+1}-\boldsymbol{w}_{i}\right)+\frac{\delta t^{+}}{2}\left(\boldsymbol{v}_{i+1}^{+}-\boldsymbol{v}_{i}^{+}\right)^{2},
$$

with ${ }^{+}$indicating normalized quantities, and $P e$ the Péclet number defined by:

$$
P e=\frac{\zeta b^{2} \dot{\varepsilon}}{k_{B} T},
$$

where $\dot{\varepsilon}$ is the characteristic extensional strain rate, such that $\boldsymbol{K}=\dot{\boldsymbol{\varepsilon}} \boldsymbol{K}^{+}$. The Péclet number quantifies, at the scale of a single rod, the competition between the shuffling Brownian motion and the macroscopic fluid drive.

The Weissenberg number, $W i$, is another desirable characteristic variable needed to describe the dynamics of polymers in solution. It is defined by the product of the strain rate $\dot{\varepsilon}$ times the longest relaxation time of the polymer. Using extensive simulations, Doyle et al. (1997) proposed the scaling relation for the free-draining bead-rod model:

$$
W i=0.0142 N^{2} P e .
$$

We note that the coefficient 0.0142 is close to $1 /\left(6 \pi^{2}\right) \approx 0.0169$ from the Rouse model.

A final remark has to be made about the free-draining nature of the model. Internal hydrodynamic interactions (HI) and excluded volume effects (EV) are known to have a significant impact on the dynamics of polymers in solution (Teraoka 2002). Integrating these effects in simulations greatly increases the algorithm complexity and computational time. As a result, $\mathrm{HI}$ and EV have long been confined to the simulations of chains with very limited number of rods. Although notable improvements have been made in this field over the past few years (Banchio \& Brady 2003; Geyer \& Winter 2009; Schmidt et al. 2011; Saadat \& Khomami 2015; Moghani \& Khomami 2017), we decided not to incorporate HI and EV in our simulations for several reasons. First, the flexible and high molecular weight polymers of interest in this paper require large numbers of rods (over 1000), which still makes HI and EV computationally prohibitive. Second, previous simulations of the scission of moderate length chains have shown that the rupture dynamics do not depend strongly on the existence of $\mathrm{HI}$ and EV (López Cascales \& García de la Torre 1991; Knudsen et al. 1996b; Sim et al. 2007). This is due to the fact that the HI and EV coupling is weak near the fully stretched configuration, and in cases of practical importance, the chains are indeed mostly unravelled by the extensional flow before being broken.

\subsection{Adaptive time stepping and rod coarse-graining}

Even without modelling $\mathrm{HI}$ and EV effects, the simulation of bead-rod chains above $10^{4}$ rods over a few milliseconds of physical time requires substantial computing resources. It can be possible to study a single molecule but this approach quickly becomes unreasonable for a population of a few hundred. Two modifications have thus been made to the standard bead-rod algorithm in order to enhance its throughput. 
First, we implemented an adaptive time stepping approach. The simulation of each chain starts with a normalised time step of $\delta t^{+}=10^{-3}$, which is sufficiently fine for unravelled chain motion. However, as the chain catches more of the flow gradient, the time step has to be reduced, otherwise the iterative solver of equation 3.3 does not necessarily converge. This is done automatically by recursively halving the time step until convergence is reached. In this manner, the finer time dynamics are computed only when required.

The second modification is based on the consideration that the tension in a straight segment is essentially a parabola (Sim et al. 2007) and so can be described by a relatively small subset of rods. We performed this approximation by a coarse-graining of the chain at locations where the internal tension greatly exceeds the Brownian force, here: $g_{i}^{+}>100$. Contiguous rods meeting this criterion are merged and the friction coefficients $(\zeta)$ of remaining beads are increased accordingly. Typical speed-up factors of 20 to 140 can be achieved by allowing 6 recursive levels of rod merging.

\subsection{Chain scission hypothesis}

In the bead-rod model, we assume that a chain breaks if there is a rod where the internal tension reaches the value, $g_{c}$, reflecting the breaking of a backbone bond. Typically reported values for a C-C single bond span from $2.6 \mathrm{nN}$ to $13.4 \mathrm{nN}$, whether it is calculated from experiments or ab initio simulations, and possibly depend, though weakly, on the force loading rate (Odell \& Keller 1986; Grandbois et al. 1999; Beyer 2000; Stauch \& Dreuw 2016). As explained in detail by Sim et al. (2007), because of the discrete random forces $\boldsymbol{w}^{+}$, solving equation 3.4 gives normalized tensions that fluctuate with variance scaling as $\mathscr{O}\left(N / \delta t^{+}\right)$. If we want to set a threshold criterion for the internal tension, it is necessary to filter any unphysical fluctuations. Several methods have been proposed, requiring additional hypotheses or substantial post-processing (Sim et al. 2007; Schieber \& Obasanjo 2005). Here, we choose a simpler approach. We don't remove completely the time-step dependent fluctuations, but keep them within the typical error margin of $g_{c}$. To achieve this, the value of the tension is time-averaged over $p$ time steps. It follows that the variance of fluctuations is reduced to $\mathscr{O}\left(N /\left(p \delta t^{+}\right)\right)$. We will see that the normalised rupture force, $g_{c}^{+}$, is of the order of $10^{3}$, over which we can assume a $10 \%$ relative error. Therefore, by choosing $p>10^{-4} \mathrm{~N} / \delta t^{+}$, we arrive at a reasonably smoothed estimate for the internal tension. On the other hand, $p$ should be kept as low as possible in order to keep track of the transient dynamics.

\subsection{Setting dimensions of the bead-rod model}

Two parameters are essential for setting the dimensions of the bead-rod model. First, the Kuhn length is assessed from tabulated data (Brandrup et al. 1999):

$$
b=\frac{C_{\infty} \ell}{0.84}
$$

where $C_{\infty}$ is a rigidity constant and $\ell$ the mean length of a backbone bond. It is then possible to know the number of rods required to represent a given molecular weight, $M$ :

$$
N=\frac{M}{M_{b}}
$$

where $M_{b}$ is the molecular weight of a single rod, given by

$$
M_{b}=\frac{M_{0} C_{\infty}}{0.84^{2} i}
$$

with $M_{0}$ the monomer molecular weight and $i$ the number of backbone bonds per monomer. The second important physical parameter is the friction coefficient of the beads. It is usually 
assessed so that the longest relaxation time of the chain matches the Zimm relaxation time of the polymer, $\tau_{Z}$ (Doyle et al. 1997). According to equation 3.6:

$$
\zeta=\frac{k_{B} T \tau_{Z}}{0.0142 N^{2} b^{2}}
$$

The Zimm time can be computed from:

$$
\tau_{Z}=\frac{[\eta] \eta_{s} M}{R T}
$$

with $[\eta]$ the intrinsic viscosity, $\eta_{s}$ the viscosity of the solvent, and $R$ the gas constant. In turn, $[\eta]$, which is a function of the molecular weight and solvent properties, is tabulated through the use of the Mark-Houwink-Sakurada law:

$$
[\eta]=K M^{a}
$$

where $K$ and $a$ are constants depending on the solvent and on temperature.

The Zimm time is relevant to the polymer dynamics in its coil state. We see from equations 3.11 and 3.12 that the scaling with the molecular weight is $M^{a+1}$, quite different from the free-draining $M^{2}$ (equation 3.6). Yet, near full extension, the hydrodynamic shielding of the chain vanishes, and a correct friction coefficient has to be chosen. We can assess this coefficient by taking its value for a single Kuhn step:

$$
\zeta_{b}=\frac{\eta_{s} K M_{b}^{a+1}}{0.0142 \mathscr{N}_{A} b^{2}},
$$

with $\mathscr{N}_{A}$ the Avogadro number. Table 1 summarises experimentally determined parameters for two polymer solutions: Poly(methyl methacrylate) (PMMA) in ethyl acetate, and PEO in water. Molecular constants are taken from Brandrup et al. (1999).

\subsection{Tension in a fully stretched chain}

The internal tension in a fully stretched chain will be the starting point of our macroscopic formulation. By fully stretched, we mean that the rods are perfectly aligned with each other. If, in addition, the chain is stretched in a uni-axial extensional flow and aligned with the strain axis, it is known that the internal tension has a parabolic shape with a maximum at the centre of the molecule, given by (Sim et al. 2007):

$$
g_{\max }=\frac{\zeta \dot{\varepsilon} L^{2}}{8 b}
$$

with $L$ the contour length of the molecule. This expression can be generalized to an arbitrary flow field where the chain is still fully stretched but not necessarily aligned with the elongation:

$$
g_{\max }=\frac{\zeta \nabla \boldsymbol{u}: \boldsymbol{R} \boldsymbol{R}}{8 b} .
$$

This expression is ensemble-averaged to get:

$$
\left\langle g_{\max }\right\rangle=\frac{\zeta \nabla \boldsymbol{u}: \boldsymbol{A}}{8 b}
$$

where we have introduced the conformation tensor $\boldsymbol{A}$ defined in section 2. Equation 3.16 is an expression of the average maximum internal tension as a function of macroscopic quantities. Albeit derived for a steady state elongation, we will see in the following that it provides a good approximation in transient and arbitrary flows. 
Table 1: Dimensioning parameters for the bead-rod model (room temperature).

\begin{tabular}{lcc}
\hline & PMMA & PEO \\
\cline { 2 - 3 } Solvent & ethyl acetate & water \\
$\eta_{s}(\mathrm{mPa} . \mathrm{s})$ & 0.42 & 1.0 \\
$K \times 10^{9}\left(\mathrm{~m}^{3} / \mathrm{g}\right)$ & $21^{*}$ & 156 \\
$a$ & $0.64^{*}$ & 0.50 \\
$C_{\infty}$ & 9.0 & 3.8 \\
$\ell(\AA)$ & 1.5 & 1.4 \\
$b(\mathrm{~nm})$ & 1.6 & 0.63 \\
$M_{b}(\mathrm{Da})$ & 638 & 79 \\
$k_{B} T / b(\mathrm{pN})$ & 2.6 & 6.5 \\
$g_{c}(\mathrm{nN}) \mathrm{Beyer}(2000)$ & 4.7 & 5.0 \\
$g_{c}^{+} \times 10^{-3}$ & 1.8 & 0.77 \\
$\zeta_{b} \times 10^{11}(\mathrm{~N} . \mathrm{s} / \mathrm{m})$ & 1.6 & 3.2 \\
$\zeta b^{2} /\left(k_{B} T\right)(\mathrm{ns})$ & 9.8 & 3.1 \\
Pe for $\dot{\varepsilon}=10^{6} \mathrm{~s}{ }^{-1}$ & 0.010 & 0.0031 \\
& \multicolumn{2}{c}{} \\
& for $M=10^{6} \mathrm{Da}$ \\
$L(\mu \mathrm{m})$ & 2.5 & 8.0 \\
$N$ & 1567 & 12658 \\
$P e_{c}$ & 0.0059 & $3.8 \times 10^{-5}$ \\
\hline$*$ From in-house viscometric measurement &
\end{tabular}

\subsection{Reaction rate}

López Cascales \& García de la Torre (1992) studied the chemical kinetics of chain fracture under a sudden elongational flow. They employed two bead-spring models, each with a specific elastic function and maximum stretching energy for the springs. They found that the reaction kinetics can be described by two regimes: a first period of time when the chains align with the flow and start unravelling; then a second step when the chains break following a first order reaction rate. They analysed the scaling of the reaction constant with the strain rate and the number of beads.

In practical flows however, the extensional rate varies either when the flow is transient, or when the molecules travel through regions of different flow gradients. For example, let us consider a population of random coils subject to an increasing Péclet number (increasing strain rate). According to equation 3.14, we can define a critical Péclet for the onset of chain rupture:

$$
P e_{c}=\frac{8 g_{c}^{+}}{N^{2}} .
$$

For $W i>1 / 2$, the chains unravel, but don't break as long as $P e<P e_{c}$. If they stay in this situation during at least a few $P e^{-1}$ (in normalized time units), they unravel completely and the population is composed of homogeneous stretched chains. The tension in each chain then differs from $\left\langle g_{\max }\right\rangle$ only by thermal fluctuations. Then, if at this stage, $P e \geqslant P e_{c}$, all the chains break simultaneously regarding the macroscopic time scale. On the other hand, if $P e$ becomes greater than $P e_{c}$ in less than $P e^{-1}$ (which is the case for the sudden elongation in López Cascales \& García de la Torre 1992 and contraction flows in general), only the chains that have significant unravelled portions break. The rate at which the population of intact chains decreases depends on the unravelling dynamics of individual molecules.

For a sudden elongation, we reproduced the numerical experiment of López Cascales \& 

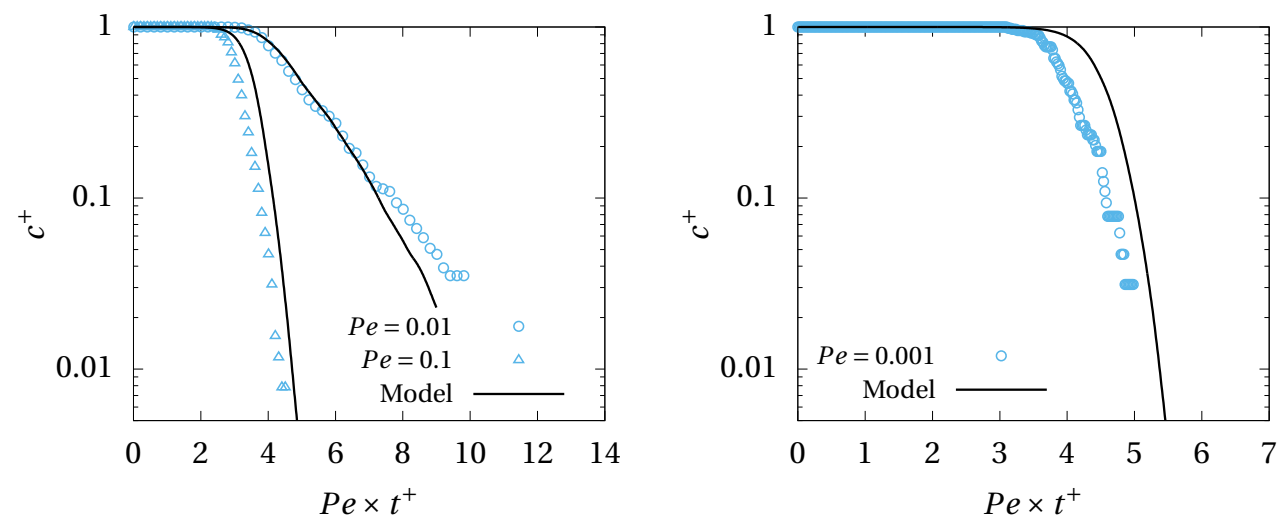

Figure 3: Bead-rod model simulation of the evolution of the relative concentration of intact chains subject to a sudden extensional flow of various strength. Left: PMMA ( $N=1567, P e_{c}=$ $\left.5.9 \times 10^{-3}\right)$, right: $\mathrm{PEO}\left(N=12658, P e_{c}=3.8 \times 10^{-5}\right)$.

García de la Torre (1992) but with the free-draining bead-rod model presented above. We use the parameters of table 1 for the PMMA 1 MDa and the PEO 1 MDa solutions. Results for various strain rates are reported in figure 3. For every strain rate, 256 molecules with random initial configurations are simulated and chain scission events recorded. The normalized concentration $c^{+}$of initial chains is plotted against the total strain $P e \times t^{+}$. At long times (equivalently at high strain), an exponential decrease in $c^{+}$is found, suggesting again a first order scission rate. The rate constant is $P e / 2$ if the critical tension is close to the tension in the fully stretched chain, in other words, $P e \sim P e_{c}$ (chains unravel first, then break), and higher if $P e \gg P e_{c}$. More specifically, our bead-rod simulations show that, when $P e$ is close to $P e_{c}$, the concentration $c^{+}$is fairly represented by the functional form:

$$
c^{+}=\left(1-\lambda^{2}\right)^{\frac{P e}{P e_{c}}},
$$

where $\lambda=\operatorname{tr}(\boldsymbol{A}) / L^{2}$ is the normalised extension of the chains. A convenient way of estimating $P e$ through $\dot{\varepsilon}$ in arbitrary flow is $\dot{\varepsilon} \approx \nabla \boldsymbol{u}: \boldsymbol{A} / \operatorname{tr}(\boldsymbol{A})$. The ratio $P e / P e_{c}$ can then also be put in terms of the internal tension, according to equations 3.16 and 3.17: $P e / P e_{c} \approx\left\langle g^{+}\right\rangle /\left(\lambda g_{c}^{+}\right)$. The first order kinetic coefficient, $k$, defined by $k=-\frac{\mathrm{d}}{\mathrm{d} t} \ln c^{+}$is then:

$$
k=-\frac{\mathrm{d}}{\mathrm{d} t}\left[\frac{\left\langle g^{+}\right\rangle}{\lambda g_{c}^{+}} \ln \left(1-\lambda^{2}\right)\right] .
$$

Equation 3.19 gives a general model for chain scission, complemented with the following assumptions:

(i) No recombination of chains: $k \geqslant 0$. In particular, $k=0$ for relaxing chains with $\mathrm{d}\left\langle g^{+}\right\rangle / \mathrm{d} t<0$.

(ii) No scission occurs below the threshold Péclet number: $k=0$ for $P e<P e_{c}$.

Note that we restrict the model to the scission of the initial chains only. If strain rates are sufficiently high, scission products might break in turn, as demonstrated by experiments and simulations (Odell \& Keller 1986; Sim et al. 2007).

\subsection{CFD model}

In the previous subsections, we obtained closed forms of the ensemble-average peak tension in chains and of the scission kinetic rate as functions of the conformation tensor. 


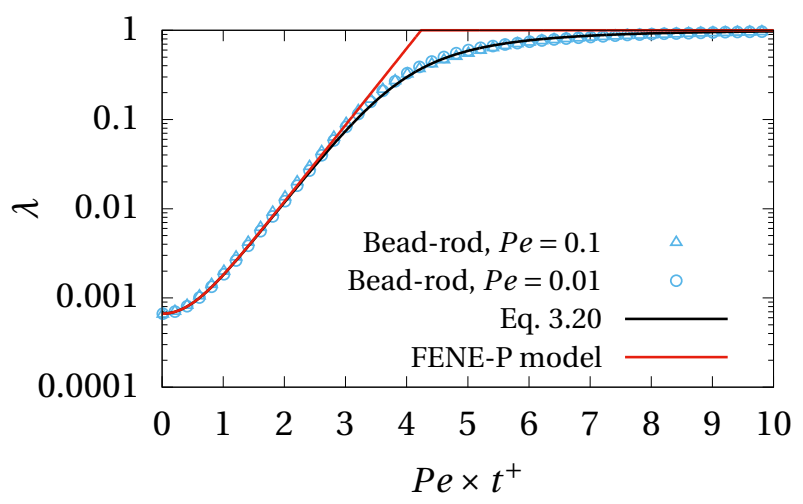

Figure 4: Unravelling dynamics of PMMA chains $(N=1567)$ during a sudden elongation.

What remains to be chosen is then the constitutive equation to solve $\boldsymbol{A}$ at the macroscopic scale. Here, we employ an upper-convected model adapted from Hinch (1994):

$$
\stackrel{\nabla}{\boldsymbol{A}}=-\max \left[0,\left(\frac{7}{2}-\frac{3}{2} \lambda\right) \nabla \boldsymbol{u}: \frac{\boldsymbol{A}}{L^{2}}\right] \boldsymbol{A}-\frac{\boldsymbol{A}-\frac{R_{0}^{2}}{3} \boldsymbol{I}}{\tau},
$$

where $\stackrel{\nabla}{\boldsymbol{A}}=\frac{\mathrm{d}}{\mathrm{d} t} \boldsymbol{A}-\boldsymbol{\nabla} \boldsymbol{u}^{T} \boldsymbol{A}-\boldsymbol{A} \boldsymbol{\nabla} \boldsymbol{u}$ is the upper-convected derivative of $\boldsymbol{A}, R_{0}$ is the ensembleaveraged end-to-end distance of the unperturbed chains, and $\tau$ is a relaxation time. The first term of the right-hand side accounts for both the unravelling dynamics and finite extensibility of the chains. Although this term has the form of an internal viscosity, it is not due to any frictional process, but rather to the complex topological evolution of unfolding kinks (Hinch 1994). When the inner product $\boldsymbol{\nabla} \boldsymbol{u}: \boldsymbol{A}$ is negative, the chains are contracting, and this effective internal viscosity has to be set to zero. From our uniaxial extension simulations of bead-rod chains, we found that the $(7 / 2-3 / 2 \lambda)$ factor is necessary to ensure that the conformation converges exponentially towards fully stretched chains at the correct rate of $\dot{\varepsilon} / 2$. The second term of the right-hand side is an exponential relaxation in the absence of flow gradient. The relevance of Eq. 3.20 can be tested by plotting $\lambda$ as a function of time during the sudden elongation simulations of the previous subsection. Results are shown in figure 4, where another common constitutive equation, the FENE-P model (Finitely Extensible Nonlinear Elastic with Peterlin closure, Bird \& Wiest 1995) is also plotted. In particular, we see that the internal viscosity term is necessary to capture the slowdown of unravelling towards the steady state.

Effects such as hydrodynamic interactions, concentration or multi-mode relaxation are neglected here, but could be incorporated in more complex or empirical models (Lielens et al. 1999; Ilg et al. 2010). In addition, we assume very dilute concentrations, so that the viscous stress tensor is not affected by the presence of polymers. This is justified as an initial approach by the fact that the scission rate depends on local flow gradients, regardless of whether the shape of the flow was influenced by non-Newtonian effects or not. By doing so, the velocity field of the fluid is not coupled with the conformation tensor and can be solved by a standard method.

The scission model was implemented using the C++ library OpenFOAM (Weller et al. 1998; Favero et al. 2010). The CFD solver can be summarised as follows. For each time step:

(i) The velocity $\boldsymbol{u}$ and pressure fields are solved using the PISO algorithm (OpenFOAM standard solver); 


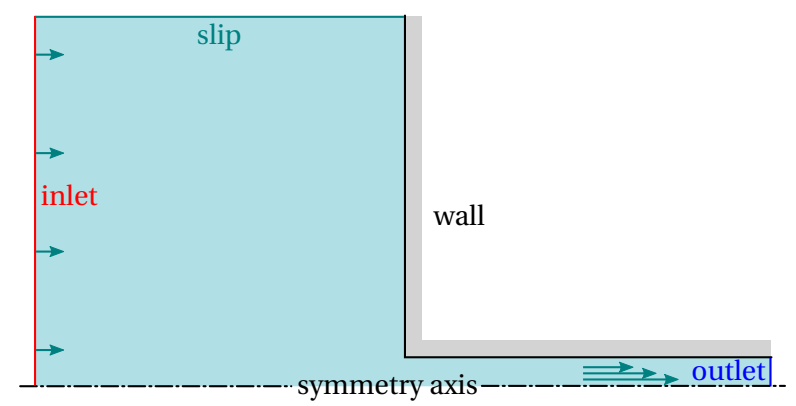

Figure 5: Geometry of the simulation domain.

(ii) $\boldsymbol{A}$ is solved (equation 3.20);

(iii) $\langle g\rangle$ and $k$ are updated (equations 3.16 and 3.19);

(iv) $c$ is solved (convected scalar with a first order mass sink).

In the next section, we present simulation results and discuss the consistency of the multiscale approach.

\section{Results and discussion}

\subsection{Contraction flow}

Mechanical scission of polymer chain has been observed in various flow patterns such as cross-slot flows, sonication or contraction flows (May \& Moore 2013). Contraction flows in particular have been widely studied and represent one of the most common source of extensional strain in fluid processes such as flows through nozzles in inkjet printing, or extrusion in fused deposition modelling, and fluid dispensing with needles. Their characteristics are a short residence time in the extensional zone for molecules following the centre line, and a mix of shear and elongation close to the wall, with smaller velocities and thus longer residence times. The consequence of this heterogeneity is that molecules experience a variety of strain rates and therefore undergo different behaviours. For example, it has been found in experiments that multiple passes are required to break a significant number of chains (Harrington \& Zimm 1965; Buchholz et al. 2004; Clay \& Koelling 1997).

Several groups have modelled the flow of polymer chains, including DNA, through a contraction making use of Brownian dynamics simulations (Knudsen et al. 1996a,b; Sim et al. 2007). However, most of the emphasis was put on molecules that follow the centreline only, disregarding scission events near the walls. Moreover, in some geometries, the high Reynolds numbers in the contraction suggest that the flow becomes turbulent downstream, which could enable chain scission in turbulent bursts, not captured by the simulation.

For our study, we model an axisymmetrical sharp contraction where the outlet capillary diameter is $80 \mu \mathrm{m}$, represented in figure 5. The fluid velocity, as well as the conformation tensor, are prescribed and uniform at the inlet boundary, zero gradient otherwise, except at the wall where the velocity is zero. The velocity value sets the flow rate of the simulation case and the conformation tensor is that of an unperturbed population of chains. The pressure is set to zero at the outlet (we are not considering cavitation phenomena), and zero gradient otherwise. It is thus assumed that the flow is fully developed at the outlet. The mesh is composed of 72000 rectangular cells and can be downloaded as a supplementary material. 
Table 2: Simulation cases and global result.

\begin{tabular}{|c|c|c|}
\hline Flow rate $(\mathrm{ml} / \mathrm{h})$ & \multicolumn{2}{|c|}{ Reynolds number Conversion yield (\% } \\
\hline \multicolumn{3}{|c|}{ PMMA (1 MDa) in ethyl acetate } \\
\hline 27.0 & 250 & 0 \\
\hline 54.0 & 500 & 0.043 \\
\hline 109 & 1000 & 4.1 \\
\hline 162 & 1500 & 8.1 \\
\hline 216 & 2000 & 12 \\
\hline 270 & 2500 & $17^{*}$ \\
\hline 323 & 3000 & $23^{*}$ \\
\hline 377 & 3500 & $30^{*}$ \\
\hline \multicolumn{3}{|c|}{ PEO (1 MDa) in water } \\
\hline 5.65 & 25 & 0.94 \\
\hline 11.3 & 50 & 8.1 \\
\hline 17.0 & 75 & 23 \\
\hline 22.6 & 100 & 39 \\
\hline 28.3 & 125 & 51 \\
\hline 33.9 & 150 & 61 \\
\hline 56.5 & 250 & 97 \\
\hline 113 & 500 & 100 \\
\hline
\end{tabular}

The Reynolds number in the contraction is defined by:

$$
R e=\frac{4 \rho Q}{\pi \eta D},
$$

where $\rho$ and $\eta$ are the solvent density and viscosity, $Q$ is the volumetric flow rate, and $D$ is the diameter of the outlet. To trigger chain scission, $Q$ has to be high in order to generate large extensional rates. On the other hand, $R e$ has to be kept limited to avoid turbulence. In our simulations, inertial instabilities usually appear above a Reynolds number of 2500 . Simulations are done for the two types of polymer solutions (PMMA in ethyl acetate and PEO in water) and various flow rates. The conversion yield, defined by the ratio of the flux of broken molecules over the total solute flux at the outlet, is recorded at the steady state. Results are reported in table 2. For the PMMA solution, simulations suggest that it is not possible to get $100 \%$ of conversion yield with a single pass in a laminar flow regime. However, a nearly complete degradation should be obtained for the PEO solution at a Reynolds number of 250 . In the next paragraph, we turn our attention to the local scission rate.

\subsection{Scission zones}

In order to describe the location of scission events, we look at two situations: the PMMA solution at $R e=2000$ and the PEO at $R e=100$. Results for a steady state flow are reported in figure 6. For the PMMA solution, the map of the scission rate, $k$, (fig. 6a) shows that the polymer breaks close to the sharp corner, upstream from the contraction. The maps of the conversion factor, $1-c^{+}$, which is the proportion of broken chains (fig. $6 \mathrm{~b} \& \mathrm{c}$ ) confirm that a very large proportion of chains that flow through this region near the edge are broken. No rupture occurs along the centreline. By contrast, the PEO solution displays a different pattern. The scission zone spans across the entire entrance of the contraction (fig. 6d), which means some of the molecules flowing through the centre are damaged. This can be seen on the conversion maps (fig. 6e\&f), where approximately $20 \%$ of the chains are broken through 
PMMA in ethyl acetate

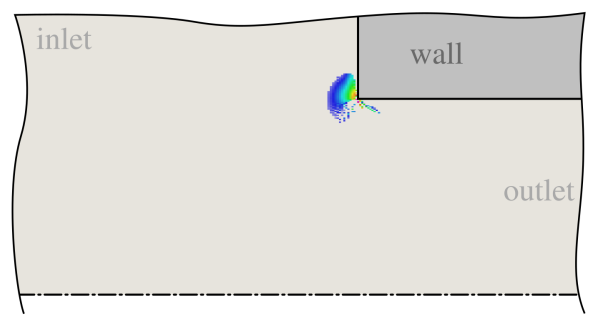

$\begin{array}{llllllll}1.000 e+052 e+5 & 5 e+5 & 1 e+6 & 2 e+6 & 5 e+6 & 1 e+7 & 2 e+7 & 5 e+71.000 e+08\end{array}$

(a) Scission rate $k\left(\mathrm{~s}^{-1}\right)$ (zoom)

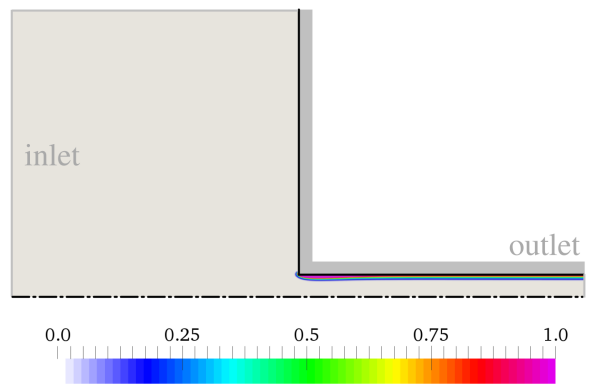

(b) Local conversion (full view)

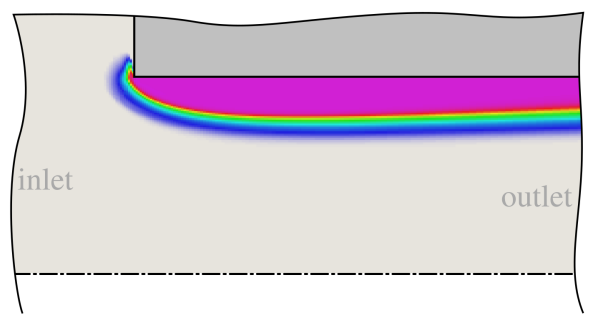

(c) Local conversion (zoom)
PEO in water

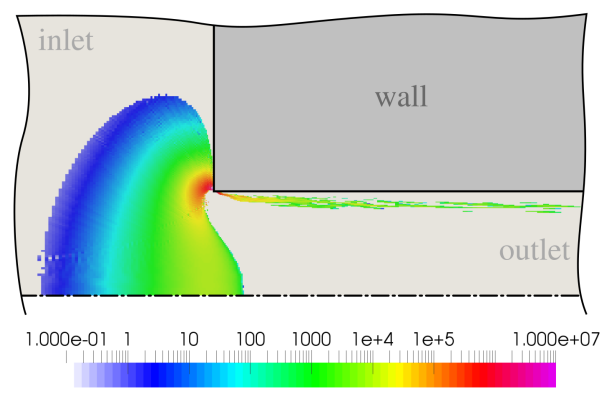

(d) Scission rate $k\left(\mathrm{~s}^{-1}\right)($ zoom)

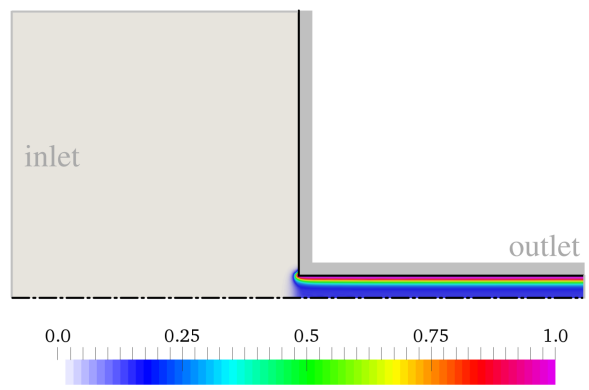

(e) Local conversion (full view)

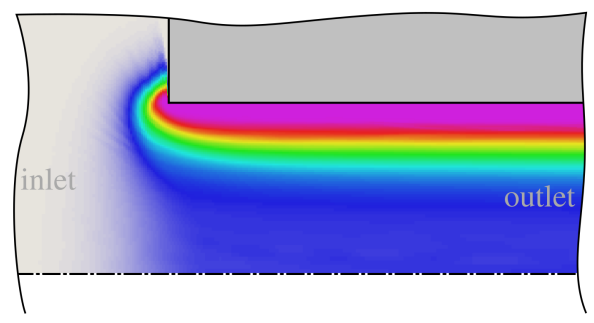

(f) Local conversion (zoom)

Figure 6: CFD results maps. Left: PMMA in ethyl acetate at $R e=2000(216 \mathrm{ml} / \mathrm{h})$; right: PEO in water at $R e=100(22.6 \mathrm{ml} / \mathrm{h})$. (a\&d) Scission rate $\left(\mathrm{s}^{-1}\right)$; (b,c\&d,e) Conversion (scission) of initial polymer chains (shared scale bar).

the centreline. Like in the case of PMMA, every PEO molecules flowing near the sharp corner undergo chain scission. 


\subsection{Comparison with the molecular model}

In order to discuss the consistency of the multiscale approach, the ensemble-averaged quantities given by both the CFD and bead-rod model are compared in the case of the PMMA solution at $R e=2000$, along three streamlines. The paths and their starting points (time 0 ) are shown in figure 7 . The corner streamline is chosen very close to the sharp edge so that a large portion of chains are broken; limit is a streamline where only a very limited amount of chain scissions occur; and centre is the centreline. The strain rate tensor extracted along the streamline with the velocity of a massless particle is given as input for the beadrod model. 256 runs are done along the same particle path, each with a random initial configuration for the chain. This method provides ensemble-averaged quantities such as the mean normalised extension of the chain $\lambda$, and the ensemble-average force in the chains $\langle g\rangle$, and ultimately the proportion of broken chains.

Results are shown in figure 8 . Plots $(8 \mathrm{a}-\mathrm{c})$ compare $\lambda$ in the two models. For the corner and limit trajectories, the agreement is good upstream from the capillary, but the bead-rod model exhibits a larger extension in the tube. The agreement is evident along the centreline (8c). This general behaviour can be explained by the fact that the constitutive equation 3.20 has been optimised to fit purely elongational flows, such as along the symmetry axis of the contraction, whereas significant vorticity is developed near the edge, reaching the characteristics of a shear flow. Another cause of deviation can be the numerical diffusion introduced by the discretisation scheme, which is likely to be particularly severe near the sharp edge.

The internal tension in the chains $\langle g\rangle(8 \mathrm{~d}-\mathrm{f})$ displays three different regimes in each streamline. Far upstream from the contraction, the tension from the bead-rod model starts from a lower bound about $0.02 \mathrm{nN}$, corresponding to thermal fluctuations only, whose impact is not present in the CFD model. Then the tension reaches a peak where both models are in agreement, and finally the models diverge downstream of the contraction. This difference can assumed to be caused again by vorticity, and also by a distinct tension dynamics in recoiling chains. To sum up, equation 3.16 provides a good macroscopic description of the bead-rod model for nearly unravelled chains under tension, which is often when mechanochemistry will occur.

Both models are in very good agreement ( $3 \%$ difference) regarding the conversion factor, and predict about $63 \%$ of chain scission along the corner streamline, as seen in figure (8g). The CFD value is not monotonic but fluctuates due to interpolation between cells. On the other hand, the models do not match along the limit line (figure 8h) for the following reasons:

(i) Low mesh resolution: in the CFD model, scissions occur in a small number of cells (figure 6a), hence a too coarse description of the scission rate along this streamline.

(ii) Numerical diffusion: the conversion factor diffuses towards cells with less scission. Nevertheless, this discrepancy should affect only a thin region of the simulation domain, and in particular, is not likely to disturb qualitative considerations (where the chains break and the influence of the device geometry), or global quantities that we now present below. 


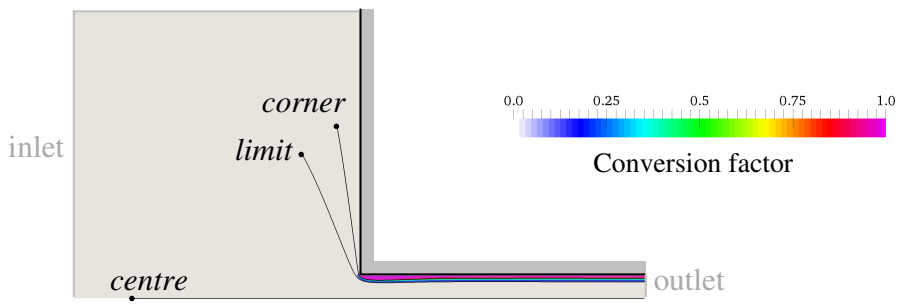

Figure 7: Three streamlines used for comparison with the bead-rod model, shown on top of the conversion factor (PMMA in ethyl acetate at $R e=2000$ ).

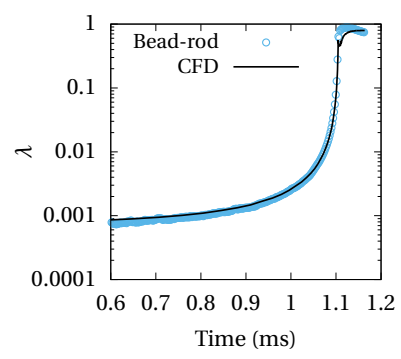

(a) Corner

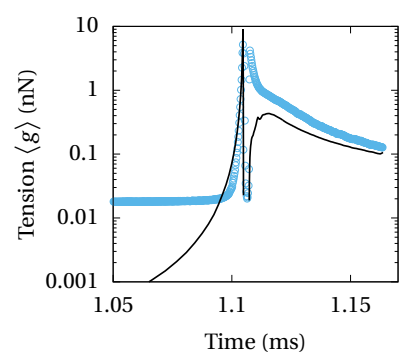

(d) Corner

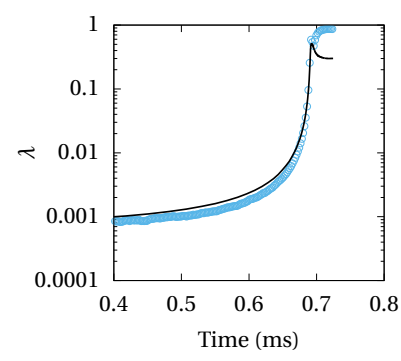

(b) Limit

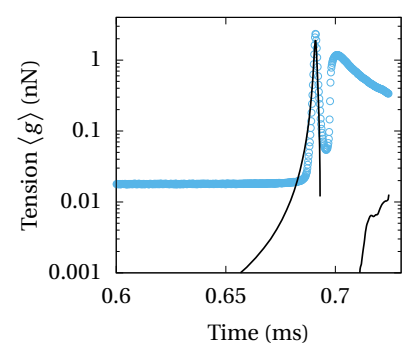

(e) Limit

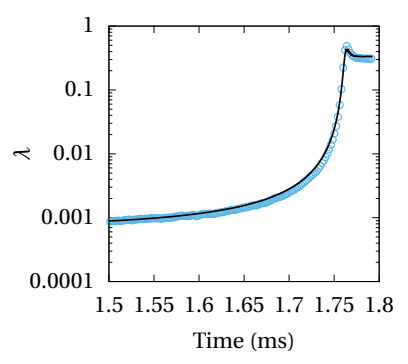

(c) Centre

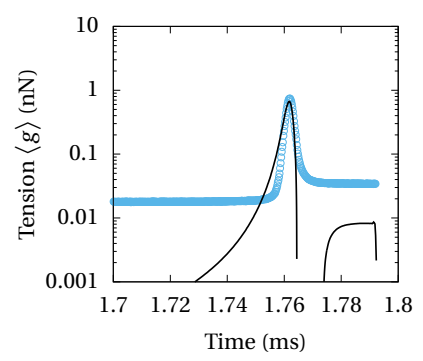

(f) Centre

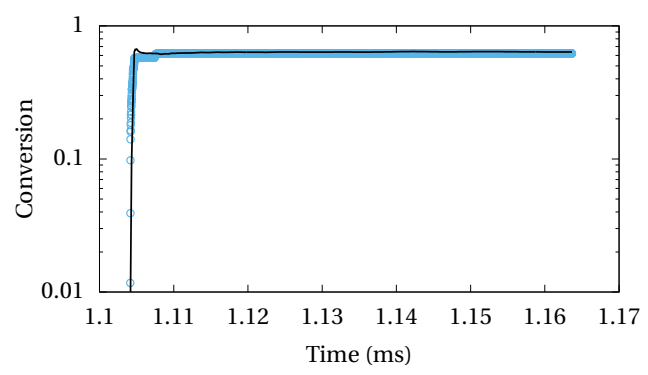

(g) Corner

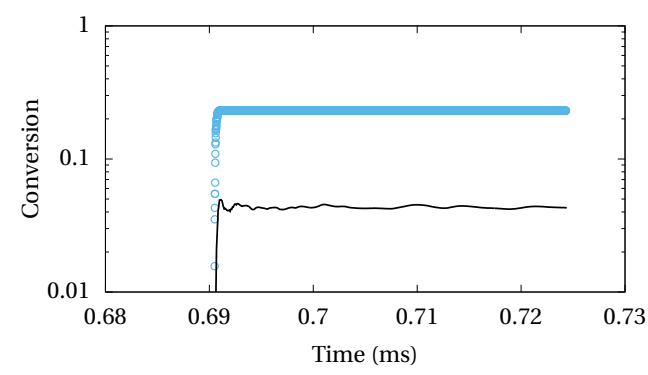

(h) Limit

Figure 8: Comparison between the bead-rod and CFD models (only the end of the trajectories are shown): (a-c) Normalised extension of the chains, $\lambda$, (d-f) Ensembleaveraged maximum tension $\langle g\rangle$; (g\&h) Conversion factor (no scission for the centreline in both models). 


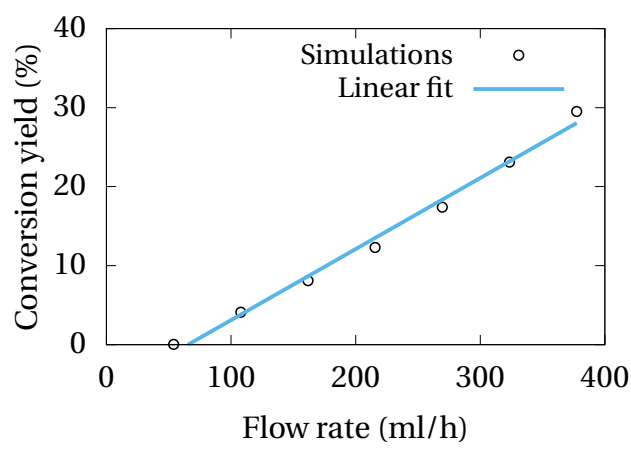

(a)

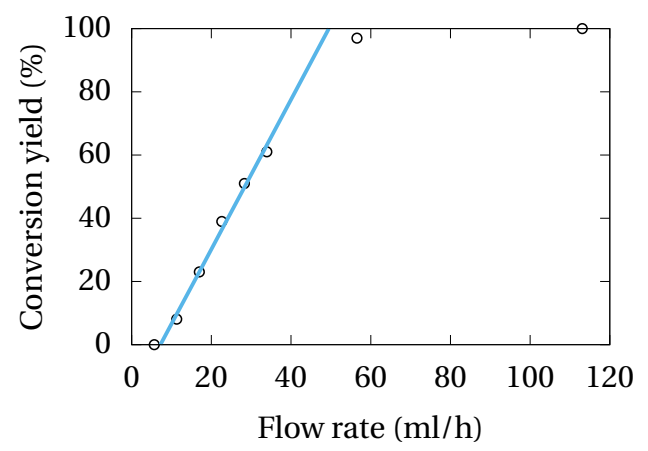

(b)

Figure 9: Conversion yield as a function of flow rate, and linear fit defining the critical flow rate; (a) PMMA in ethyl acetate; (b) PEO in water.

\subsection{Critical flow rate}

The threshold nature of polymer scission in strong flows has often been characterised by a critical elongational rate or a critical flow rate. In experiments, where measuring this threshold directly is subject to high noise, a linear interpolation of any changing properties such as the fluid viscosity or the conversion yield is made instead. The linear fit crosses the x-axis at a critical flow rate, $Q_{c}$. The simulations described in table 2 show the same phenomenon, and a linear fit is possible below a conversion of up to $30-60 \%$, as shown in figure 9. According the simulations of this $80-\mu \mathrm{m}$-diametre contraction, the critical flow rates are $Q_{c}=65 \pm 10 \mathrm{ml} / \mathrm{h}$ for $1 \mathrm{MDa} P M M A$ in ethyl acetate, and $Q_{c}=4 \pm 1 \mathrm{ml} / \mathrm{h}$ for $1 \mathrm{MDa}$ PEO in water. The rather high uncertainty on the interpolated value of $Q_{c}$ from the linear fit suggests that this method only indicates the order of magnitude of the critical flow rate.

Nevertheless, this type of curve provides a method to calibrate the model by tuning parameters (such as the bead friction $\zeta$ or the critical tension $g_{c}$ ) to fit simple scission experiments with controllable flow rate. In this way, accurate quantitative predictions could be obtained for transient and more complex flows.

\subsection{Mesh sensitivity}

The Newtonian flow around a sharp re-entrant corner has a singular velocity gradient which is improperly captured by a finite size mesh (Dean \& Montagnon 1949). Since the chain unravelling dynamics and scission rate computation depend on the velocity gradient, the robustness of the model upon mesh resolution should be tested. For this purpose, the initial mesh (78 000 cells) used so far in this paper is recursively coarsened to give three additional meshes with 18000,4500 and 1125 cells respectively. The chain scission simulations of PMMA in ethyl acetate are computed again on these meshes and results for the conversion yield (percentage of broken chains) are reported in figure 10.

Below $200 \mathrm{ml} / \mathrm{h}$, the cell resolution affects the quantitative result to a large extent, but the four meshes predict a non-zero conversion above $100 \mathrm{ml} / \mathrm{h}$; the finest mesh alone predicts a non-zero conversion at $54 \mathrm{ml} / \mathrm{h}$. On the other hand, above $200 \mathrm{ml} / \mathrm{h}$ only the coarsest mesh differs significantly from the reference, while the relative gaps with the $18 \mathrm{k}$-cell and $4.5 \mathrm{k}$-cell meshes quickly fall down to a few percents (figure 10b). This consistency at high conversion yield can be explained by the saturating first order dynamics of chain scission. The region near the corner where the mesh resolution has the most influence on the velocity gradient 


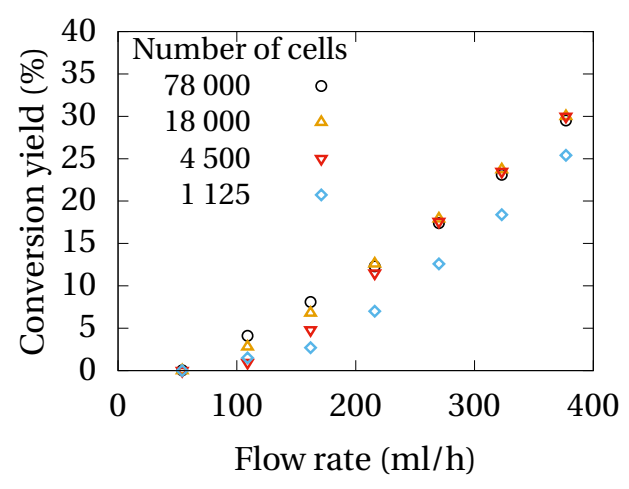

(a)

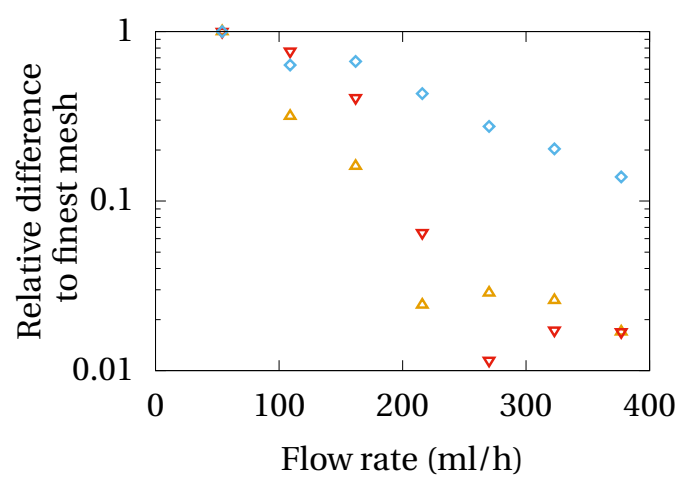

(b)

Figure 10: Mesh sensitivity results for the conversion yield of PMMA in ethyl acetate; (a) Conversion yields; (b) Relative difference to the reference mesh (78 000 cells).

magnitude is also the place where the local conversion is already close to $100 \%$. A scission rate locally increased by mesh refinement would only cause a faster conversion for chains approaching the corner, but the final yield through this region would still saturate at $100 \%$.

In summary, if very small conversion yields are the primary interest, then the effort should be placed on the accurate description of the corner, especially for comparison with manufactured channels of finite resolution. On the other hand, the model shows an acceptable robustness upon mesh coarsening for higher conversion yields, even in the presence of a sharp corner.

\section{Conclusion}

In this work, we presented a multiscale model for the scission of linear flexible polymers in laminar flows. Inspired by free-draining bead-rod model simulations, we describe the mechanochemistry as a first order reaction whose rate is given by the conformation tensor of the molecules and the flow gradient. The model in the current study is limited to very dilute polymer solutions only, although concentration can influence chain scission (Nghe et al. 2010; May \& Moore 2013). Yet, the fundamental mechanisms of this concentration effect are still unclear to a large extent. A first step to take this parameter into account could be to implement a full viscoelastic coupling between the fluid and the polymer, since increasing concentration of extended chains will have an influence on how regions of high extensional flow gradients spread out.

We presented results for steady state laminar regimes related to micro-dispensing technologies or flows through porous media. However, the model should be relevant to transient and moderately turbulent flows, as long as the viscous scale is resolved. In that case, the viscoelastic coupling seems absolutely necessary since even a fraction of polymer concentration is enough to considerably affect the onset of turbulence.

Regarding the degradation of other substances (globular biomolecules, micelles, selfassemblies, etc.), significant changes have to be made to the model to get a consistent description of the stress transfer from the flow to the object (Vasquez et al. 2007). However, the general approach should be similar: select a state variable and its constitutive equation to describe the substance conformation at the macroscopic scale, and elaborate the relationship between this state variable, the strength of the flow, and the investigated 
chemistry. In this way, new computational tools could be developed to efficiently adapt and design fluid flow processes to take mechanochemistry into account.

\section{Acknowledgement}

This work was supported by King Abdulaziz City for Science and Technology (KACST).

\section{REFERENCES}

A-Alamry, K, Nixon, K., Hindley, R., Odel, J. A. \& Yeates, S. G. 2011 Flow-Induced Polymer Degradation During Ink-Jet Printing. Macromolecul. Rapid Comm. 32 (3), 316-320.

BAnChio, A. J. \& Brady, J. F. 2003 Accelerated Stokesian dynamics: Brownian motion. J. Chem. Phys. 118 (22), 10323-10332.

Barnard, B. J. S. \& Sellin, R. H. J. 1972 Degradation of Dilute Solutions of Drag-reducing Polymer. Nature 236 (62), 12-14.

BESTUL, A. B. 1956 Kinetics of capillary shear degradation in concentrated polymer solutions. J. Chem. Phys. 24 (6), 1196-1201.

BEYER, M. K. 2000 The mechanical strength of a covalent bond calculated by density functional theory. J. Chem. Phys. 112 (17), 7307-7312.

Bird, R. B., Curtiss, C. F., Armstrong, R. C. \& Hassager, O. 1987 Dynamics of Polymeric Liquids, Volume 2: Kinetic Theory, 2nd edn. New York: Wiley-Interscience.

Bird, R. B. \& Wiest, J. M. 1995 Constitutive Equations for Polymeric Liquids. Annu. Rev. Fluid Mech. 27 (1), 169-193.

Brandrup, J., Immergut, E. H. \& Grulke, E. A. 1999 Polymer Handbook, 4th Edition. Wiley, googleBooks-ID: 5dNonQEACAAJ.

Buchholz, B. A., Zahn, J. M., Kenward, M., Slater, G. W. \& Barron, A. E. 2004 Flow-induced chain scission as a physical route to narrowly distributed, high molar mass polymers. Polymer 45 (4), $1223-1234$.

Caruso, M. M., Davis, D. A., Shen, Q., Odom, S. A., Sottos, N. R., White, S. R. \& Moore, J. S. 2009 Mechanically-Induced Chemical Changes in Polymeric Materials. Chem. Rev. 109 (11), 57555798.

Choi, H. J., Kim, C. A., Sohn, J. \& Jhon, M. S. 2000 An exponential decay function for polymer degradation in turbulent drag reduction. Polym. Degrad. Stabil. 69 (3), 341-346.

ClaY, J. D. \& Koelling, K. W. 1997 Molecular degradation of concentrated polystyrene solutions in a fast transient extensional flow. Polym. Eng. Sci. 37 (5), 789-800.

DEAN, W. R. \& MONTAGNON, P. E. 1949 On the steady motion of viscous liquid in a corner. Mathematical Proceedings of the Cambridge Philosophical Society 45 (3), 389-394.

Doyle, P. S., Shaqfen, E. S. G. \& GAST, A. P. 1997 Dynamic simulation of freely draining flexible polymers in steady linear flows. J. Fluid Mech. 334, 251-291.

Elbing, B. R., Solomon, M. J., Perlin, M., Dowling, D. R. \& Ceccio, S. L. 2011 Flow-induced degradation of drag-reducing polymer solutions within a high-Reynolds-number turbulent boundary layer. J. Fluid Mech. 670, 337-364.

Favero, J. L., Secchi, A. R., Cardozo, N. S. M. \& JaSAK, H. 2010 Viscoelastic flow analysis using the software OpenFOAM and differential constitutive equations. J. Non-Newton. Fluid 165 (23-24), $1625-1636$.

GeYeR, T. \& Winter, U. 2009 An O(N2) approximation for hydrodynamic interactions in Brownian dynamics simulations. J. Chem. Phys. 130 (11), 114905.

Grandbois, M., Beyer, M., Rief, M., Clausen-Schaumann, H. \& Gaub, H. E. 1999 How Strong Is a Covalent Bond? Science 283 (5408), 1727-1730.

Grokhovsky, S. L., Il'icheva, I. A., Nechipurenko, Dmitry Y., Golovkin, M. V., Panchenko, L. A., Polozov, R. V. \& NechipurenKo, Y. D. 2011 Sequence-Specific Ultrasonic Cleavage of DNA. Biophys. J. 100 (1), 117-125.

Harrington, R. E. \& Zimm, B. H. 1965 Degradation of Polymers by Controlled Hydrodynamic Shear1. J. Phys. Chem. 69 (1), 161-175.

Hawe, A., Wiggenhorn, M., van De Weert, M., Garbe, J. H. O., Mahler, H.-C. \& Jiskoot, W. 2012 Forced degradation of therapeutic proteins. J. Pharm. Sci. 101 (3), 895-913. 
HiNCH, E. J. 1994 Uncoiling a polymer molecule in a strong extensional flow. J. Non-Newton. Fluid pp. 209-230.

Hsieh, C.-C., Park, S. J. \& Larson, R. G. 2005 Brownian Dynamics Modeling of Flow-Induced Birefringence and Chain Scission in Dilute Polymer Solutions in a Planar Cross-Slot Flow. Macromolecules 38 (4), 1456-1468.

Ilg, P., Mavrantzas, V. \& Öttinger, H. C. 2010 Multiscale Modeling and Coarse Graining of Polymer Dynamics: Simulations Guided by Statistical Beyond-Equilibrium Thermodynamics. In Modeling and Simulation in Polymers (ed. Purushottam D. Gujrati \& Arkadii I. Leonov), pp. 343383. Wiley-VCH Verlag GmbH \& Co. KGaA, dOI: 10.1002/9783527630257.ch7.

JAMES, D. F. \& MCLAREN, D. R. 1975 The laminar flow of dilute polymer solutions through porous media. J. Fluid Mech. 70 (4), 733-752.

KANG, K., LeE, L. J. \& Koelling, K. W. 2005 High shear microfluidics and its application in rheological measurement. Exp. Fluids 38 (2), 222-232.

Knudsen, K. D., Hernández Cifre, J. G. \& García de la Torre, J. $1996 a$ Conformation and Fracture of Polystyrene Chains in Extensional Flow Studied by Numerical Simulation. Macromolecules 29 (10), 3603-3610.

Knudsen, K. D., LóPez Martinez, M. C. \& GarCía DE LA Torre, J. 1996 $b$ Fracture of DNA in transient extensional flow. A numerical simulation study. Biopolymers 39 (3), 435-444.

Larson, R. G. \& Desai, P. S. 2015 Modeling the Rheology of Polymer Melts and Solutions. Annu. Rev. Fluid Mech. 47 (1), 47-65.

Li, J., Nagamani, C. \& Moore, J. S. 2015 Polymer Mechanochemistry: From Destructive to Productive. Accounts Chem. Res. 48 (8), 2181-2190.

Lielens, G., KeUnings, R. \& LEGAT, V. 1999 The FENE-L and FENE-LS closure approximations to the kinetic theory of finitely extensible dumbbells. J. Non-Newton. Fluid 87 (2-3), 179-196.

LIU, T. W. 1989 Flexible polymer chain dynamics and rheological properties in steady flows. J. Chem. Phys. 90 (10), 5826-5842.

LuMLeY, J. L. 1969 Drag reduction by additives. Annu. Rev. Fluid Mech. 1 (1), 367-384.

LÓPEZ CASCALES, J. J. \& GARCíA DE LA TORRE, J. 1991 Simulation of polymer chains in elongational flow. Steady-state properties and chain fracture. J. Chem. Phys. 95 (12), 9384-9392.

LÓPEz CASCALES, J. J. \& GARCíA DE LA TORRE, J. 1992 Simulation of polymer chains in elongational flow. Kinetics of chain fracture and fragment distribution. J. Chem. Phys. 97 (6), 4549-4554.

Maroja, A. M., Oliveira, F. A., Ciesla, M. \& Longa, L. 2001 Polymer fragmentation in extensional flow. Phys. Rev. E63 (6), 061801.

MAY, P. A. \& MOORE, J. S. 2013 Polymer mechanochemistry: techniques to generate molecular force via elongational flows. Chem. Soc. Rev. 42 (18), 7497-7506.

Moghani, M. M. \& Khomami, B. 2017 Computationally efficient algorithms for Brownian dynamics simulation of long flexible macromolecules modeled as bead-rod chains. Phys. Rev. Fluids 2 (2), 023303.

Nghe, P., Tabeling, P. \& Ajdari, A. 2010 Flow-induced polymer degradation probed by a high throughput microfluidic set-up. J. Non-Newton. Fluid 165 (7-8), 313-322.

Odell, J. A. \& Keller, A. 1986 Flow-induced chain fracture of isolated linear macromolecules in solution. J. Polym. Sci. Pol. Phys. 24 (9), 1889-1916.

Paterson, R. W. \& Abernathy, F. H. 1970 Turbulent flow drag reduction and degradation with dilute polymer solutions. J. Fluid Mech. 43 (4), 689-710.

Pereira, A. S. \& SoARES, E. J. 2012 Polymer degradation of dilute solutions in turbulent drag reducing flows in a cylindrical double gap rheometer device. J. Non-Newton. Fluid 179-180, 9-22.

Prather, K. J., SAgar, S., Murphy, J. \& Chartrain, M. 2003 Industrial scale production of plasmid DNA for vaccine and gene therapy: plasmid design, production, and purification. Enzyme Microb. Tech. 33 (7), 865-883, wOS:000186886500001.

RALLison, J. M. \& HiNCH, E. J. 1988 Do we understand the physics in the constitutive equation? J. NonNewton. Fluid 29, 37-55.

Rathore, N. \& Rajan, R. S. 2008 Current Perspectives on Stability of Protein Drug Products during Formulation, Fill and Finish Operations. Biotechnol. Progr. 24 (3), 504-514.

REESE, H. R. \& ZIMM, B. H. 1990 Fracture of polymer chains in extensional flow: Experiments with DNA, and a molecular-dynamics simulation. J. Chem. Phys. 92 (4), 2650-2662.

Ribas-ARinO, J. \& MARX, D. 2012 Covalent Mechanochemistry: Theoretical Concepts and Computational Tools with Applications to Molecular Nanomechanics. Chem. Rev. 112 (10), 5412-5487. 
SAADAT, A. \& KHOMAmi, B. 2015 Matrix-free Brownian dynamics simulation technique for semidilute polymeric solutions. Phys. Rev. E 92 (3), 033307.

SCHIEBER, J. D. \& OBASANJo, O. 2005 On estimating stress in free-draining Kramers chain simulations using stochastic filtering. J. Non-Newton. Fluid 127 (2-3), 89-93.

SChmidt, R. R., Cifre, J. G. H. \& García de la Torre, J. 2011 Comparison of Brownian dynamics algorithms with hydrodynamic interaction. J. Chem. Phys. 135 (8), 084116.

Shendure, J. \& Ji, H. 2008 Next-generation DNA sequencing. Nat. Biotechnol. 26 (10), 1135-1145.

Sim, H. G., Khomami, B. \& Sureshrumar, R. 2007 Flow-induced chain scission in dilute polymer solutions: Algorithm development and results for scission dynamics in elongational flow. $J$. Rheol. 51 (6), 1223-1251.

Stacklies, W., Vega, M. C., Wilmanns, M. \& Gräter, F. 2009 Mechanical Network in Titin Immunoglobulin from Force Distribution Analysis. PLOS Comput. Biol. 5 (3), e1000306.

STAUCH, T. \& DREUW, A. 2016 Advances in Quantum Mechanochemistry: Electronic Structure Methods and Force Analysis. Chem. Rev. 116 (22), 14137-14180.

TeraokA, I. 2002 Polymer Solutions: An Introduction to Physical Properties, 1st edn. New York: John Wiley \& Sons.

Thorstenson, Y. R., Hunicke-Smith, S. P., Oefner, P. J. \& Davis, R. W. 1998 An automated hydrodynamic process for controlled, unbiased DNA shearing. Genome Res. 8 (8), 848-855, wOS:000075674400012.

VAN Den BRule, B., van Heel, T. \& Hulsen, M. 2011 Brownian configuration fields: A new method for simulating viscoelastic fluid flow. Macromolecular Symposia 121 (1), 205-217.

VANAPALli, S. A., CeCCIO, S. L. \& SolOMON, M. J. 2006 Universal scaling for polymer chain scission in turbulence. P. Natl. Acad. Sci. U.S.A. 103 (45), 16660-16665.

VASQUEZ, P. A., MCKINLEY, G. H. \& COOK, P., L. 2007 A network scission model for wormlike micellar solutions: I. Model formulation and viscometric flow predictions. J. Non-Newton. Fluid 144 (2), 122-139.

VIRK, P. S. 1975 Drag reduction fundamentals. AIChE J. 21 (4), 625-656.

Weller, H. G., TABor, G., JasAK, H. \& Fureby, C. 1998 A tensorial approach to computational continuum mechanics using object-oriented techniques. Comput. Phys. 12 (6), 620-631.

Wu, M. L., Freitas, S. S., Monteiro, G. A., Prazeres, D. M. F. \& Santos, J. A. L. 2009 Stabilization of naked and condensed plasmid DNA against degradation induced by ultrasounds and high-shear vortices. Biotechnol. Appl. Bioc. 53, 237-246, wOS:000268925700003. 